\title{
OBSERVATIONS OF TRANSIENT EVENTS WITH MINI-MEGATORTORA WIDE-FIELD MONITORING SYSTEM WITH SUB-SECOND TEMPORAL RESOLUTION
}

\author{
S. Karpov ${ }^{1,2,4}$, G. Beskin ${ }^{2,4}$, A. Biryukov ${ }^{5,4}$, S. Bondar ${ }^{3}$, E. Ivanov ${ }^{3}$, E. Katkova ${ }^{3}$, N. Orekhova ${ }^{3}$, \\ V. Plokhotnichenko ${ }^{2}$, A. Perkov ${ }^{3}$, V. Sasyuk ${ }^{4}$, and J. Pandey ${ }^{6}$
}

\begin{abstract}
Here we present the summary of operation of a novel 9-channel wide-field optical monitoring system with subsecond temporal resolution, Mini-MegaTORTORA, which systematically surveys the sky since 2014 at Special Astrophysical Observatory on Russian Caucasus. The system is able to observe the sky simultaneously in either wide ( $\sim 900$ square degrees) or narrow ( 100 square degrees) fields of view, either in clear light or with any combination of color (Johnson-Cousins B, V or R) and polarimetric filters installed, with exposure times ranging from $0.1 \mathrm{~s}$ to hundreds of seconds. The real-time system data analysis pipeline performs automatic detection of rapid transient events, both near-Earth and extragalactic. The objects routinely detected by Mini-MegaTORTORA also include faint meteors and artificial satellites. The imaging survey performed by Mini-MegaTORTORA also allows to look for a slower variability of various kinds of objects.
\end{abstract}

\section{RESUMEN}

Presentamos resumidamente la operación de un novedoso sistema de 9 canales para un sistema con óptica de gran campo con el que conseguimos resolución sub-segundo (Mini-MegaTORTORA). Este sistema escudriña el cielo de manera sistemática desde 2014 en el Observatorio Astrofísico Especial (SAO) en el Caúcaso ruso. El sistema es capaz de observar el cielo de manera simultánea en modo de campo amplio ( 900 grados cuadrados) o en modo estrecho ( 100 grados cuadrados), sin filtro o con filtros fotométricos (Johnson-Cousins $\mathrm{B}, \mathrm{V}$ o R) o en modo de polarización, con tiempos de exposición en el rango de 0,1s a cientos de segundos. El dataducto de análisis de los datos en tiempo real realiza detecciones automáticas de los eventos transitorios, ya sean cercanos a la Tierra o de origen cosmológico. Los objetos detectados de manera rutinaria por MiniMegaTORTORA también incluyen meteoros débiles y satélites artificiales. El mapeo del cielo realizado por Mini-MegaTORTORA también permitiría detectar variabilidad lenta en numerosas clases de objetos celestes.

Key Words: telescopes — instrumentation: miscellaneous — gamma-ray burst: general — variable stars: general

\section{INTRODUCTION}

Mini-MegaTORTORA is a novel robotic instrument developed according to the principles of MegaTORTORA multi-channel and transforming design formulated by us earlier (Beskin et al. 2010a, 2013, 2014, 2017c; Karpov et al. 2017a). It is a successor to the FAVOR (Zolotukhin et al. 2004; Karpov et al. 2005, 2010) and TORTORA (Molinari et al. 2006) single-objective monitoring instruments we built earlier to detect and characterize fast optical transients

\footnotetext{
${ }^{1}$ CEICO, Institute of Physics, Czech Academy of Sciences, Prague, Czech Republic.

${ }^{2}$ Special Astrophysical Observatory of Russian Academy of Sciences, Russia.

${ }^{3}$ Research and Production Corporation "Precision Systems and Instruments", Russia.

${ }^{4}$ Kazan Federal University, Russia.

${ }^{5}$ Moscow State University, Russia.

${ }^{6}$ Aryabhatta Research Institute of Observational Sciences (ARIES), Nainital-263002, India.
}

of various origins, both cosmological, galactic and near-Earth. The importance of such instruments became evident after the discovery and detailed study of the brightest ever optical afterglow of a gammaray burst, GRB080319B (Beskin et al. 2010c,b).

The Mini-MegaTORTORA system includes a set of nine individual channels installed in pairs on equatorial mounts. Every channel has a coelostat mirror installed before the Canon EF85/1.2 objective for a rapid (faster than 1 second) adjusting of the objective direction in a limited range (approximately 10 degrees to any direction). This allows for either mosaicking the larger field of view, or for pointing all the channels in one direction. In the latter regime, a set of color (Johnson's B, V or R) and polarimetric (three different directions) filters may be inserted before the objective to maximize the information acquired for the observed region of the sky (performing both three-color photometry and polarimetry). 
The channels are equipped with an Andor Neo sCMOS detectors having $2560 \times 2160$ pixels $6.4 \mu \mathrm{m}$ each. Field of view of a channel is roughly $9 \times 11$ degrees with angular resolution of $16^{\prime \prime}$ per pixel. The detector is able to operate with exposure times as small as $0.03 \mathrm{~s}$, in our work we use $0.1 \mathrm{~s}$ exposures providing us with 10 frames per second as on higher frame rates we are unable to process the data in real time. The dedicated dehumidifier is installed for every channel to constantly supply dry and warm air to it to prevent the dew forming inside, and to ensure the temperature is not going below zero in winter times so the electronics and mechanical parts do not freeze.

Every channel is operated by a dedicated PC which controls its hardware, acquires the images from the detector and performs the data processing. The amount of data acquired by a single channel is about $3 \mathrm{~Tb}$ in 8 hours of observations. The complex as a whole is being controlled by a separate PC.

The objectives are connected to a custom circuit boards that allow to control their focusing and aperture settings from a PCs. The dedicated autofocusing routine, based on counting the number of stars detectable on the frame, is implemented in the control software and is run periodically to ensure image quality under changing environment conditions. As a result, the FWHM of the stars as seen by MiniMegaTORTORA channels is around 2 pixels wide. The detection limit in white light for $0.1 \mathrm{~s}$ exposure is close to $11 \mathrm{mag}$, when calibrating to $\mathrm{V}$ band magnitudes.

\section{MINI-MEGATORTORA OPERATION}

Mini-MegaTORTORA started its operation in June 2014, and routinely monitor the sky since then. The observations are governed by the dedicated dynamic scheduler optimized for performing the sky survey. The scheduler works by selecting the next pointing for Mini-MegaTORTORA by simultaneously optimizing the following parameters: distances from the Sun, Moon and the horizon should be maximized, distances from the current pointings of Swift and Fermi satellites should be minimized, and the number of frames already acquired on a given sky position that night should be minimized. In this way more or less uniform survey of the whole sky hemisphere is being performed while maximizing the probability of observations of gamma-ray bursts. As an extension, the scheduler also supports the observations of pre-selected targets given by their coordinates, which may be performed in various regimes supported by Mini-MegaTORTORA (wide-field monitoring of a given region of the sky with or without filters, narrow-field multicolor imaging or polarimetry with lower temporal resolution, etc).

\subsection{Real-time transient detection}

The main regime of Mini-MegaTORTORA operation is the wide-field monitoring with high temporal resolution and with no photometric filters installed. In this regime, every channel acquires 10 frames per second, which corresponds to 110 megabytes of data per second. To analyze it, we implemented the real-time fast differential imaging pipeline intended for the detection of rapidly varying or moving transient objects - flashes, meteor trails, satellite passes etc. It is analogous to the pipeline of FAVOR and TORTORA cameras (Beskin et al. 2004; Karpov et al. 2010), and is based on building an iteratively-updated comparison image of current field of view using numerically efficient running median algorithm, as well as threshold image using running similarly constructed median absolute deviation estimate, and then comparison of every new frame with them, extracting candidate transient objects and analyzing lists of these objects from the consecutive frames. It then filters out noise events, extracts the meteor trails by their generally elongated shape on a single frame, collects the events corresponding to moving objects into focal plane trajectories, etc. (Figures 1 and 2).

Every 100 frames acquired by a channel are being summed together, yielding "average" frames with 10 s effective exposure and better detection limit. Using these frames, the astrometric calibration is being performed using locally installed Astrometry.NeT code by Lang et al. (2010). Also the rough photometric calibration is being done. These calibrations, updated every 10 seconds, are used for measuring the positions and magnitudes of transients detected by the real-time differential imaging pipeline. The "average" frames are stored permanently (in contrast to "raw" full-resolution data which is typically erased in a day or two after acquisition) and may be used later for studying the variability on time scales longer than $10 \mathrm{~s}$.

The Mini-MegaTORTORA typically observes every sky field continuousy for a 1000 seconds before moving to the next pointing. Before and after observing the field with high temporal resolution, the system acquires deeper "survey" images with 60 seconds exposure in white light in order to study the variability of objects down to 14-15 magnitude on even longer time scales; typically, every point of the 

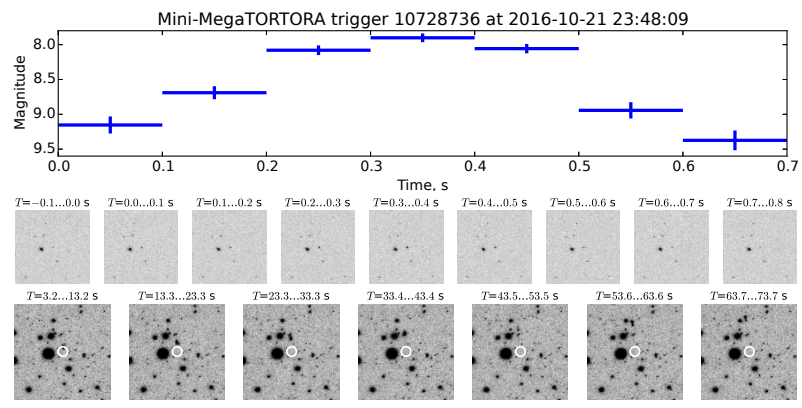

Fig. 1. Example of a rapid optical flash independently detected and followed-up by Mini-MegaTORTORA and not identified with satellites from NORAD database. Upper panel - light curve with 0.1 s temporal resolution, middle panel - corresponding detection images $\left(50^{\prime} \mathrm{x}\right.$ $50^{\prime}$ around the event), lower panel - follow-up images with $10 \mathrm{~s}$ exposures that clearly reveal a satellite slowly moving away from the flash position.
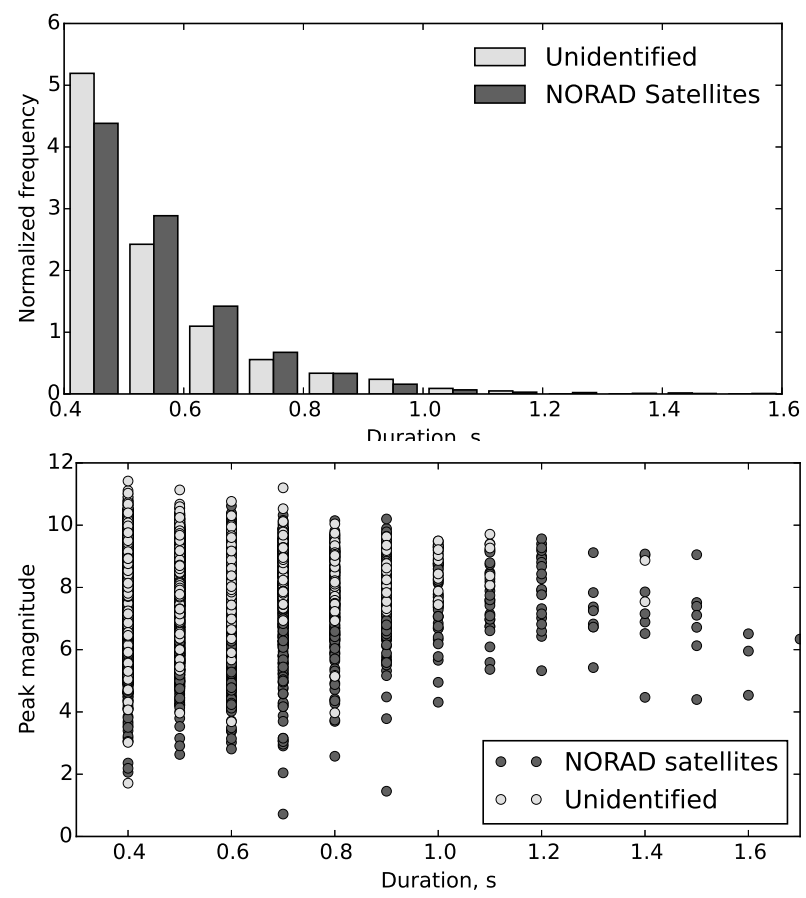

Fig. 2. Comparison of durations (upper panel) and brightness (lower panel) of rapid flashes detected by Mini-MegaTORTORA and identified/non-indentified with NORAD satellites.

northern sky is covered by one or more such images every observational night.

Mini-MegaTORTORA real-time transient detection system routinely extracts various kinds of transient from the data stream - rapid flashes, meteors, satellites etc.

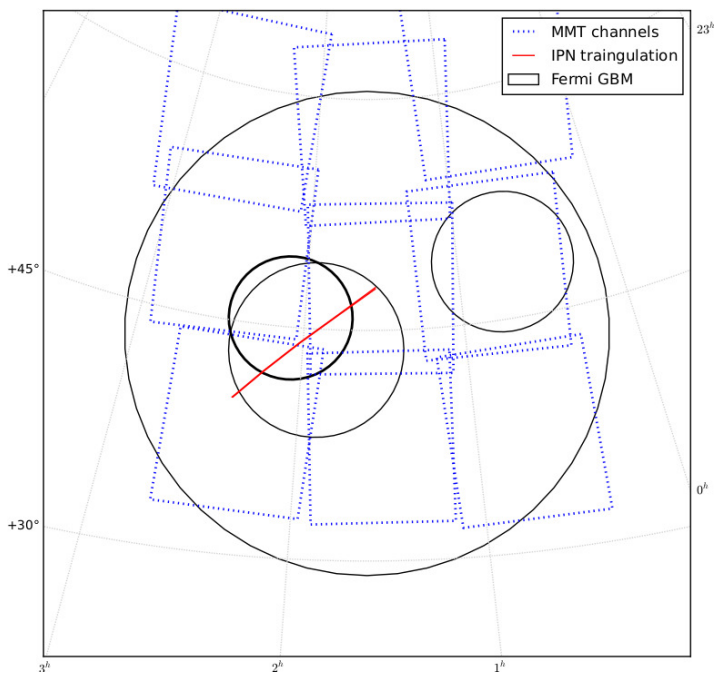

Fig. 3. The gradual improvement of GBM localization of GRB 151107B over time, and the Mini-MegaTORTORA field of view at the moment of its trigger (9 channels in wide-field regime). Most of final GBM localization, as well as IPN triangulation error box, was inside MiniMegaTORTORA field of view.

\subsection{Follow-up observations of external triggers}

Mini-MegaTORTORA also performs follow-up of Swift, Fermi and LIGO-Virgo triggers. Its large field of view, allowing for simultaneous observations of 900 sq.deg. sky regions, makes it the perfect instrument for following up events with poor localization accuracy. On the other hand, the triggers with better localizations may be observed in multicolor and/or polarimetric regimes simultaneously.

Since mid-2015, 4 of 89 Swift GRBs have been followed up in narrow-field polarimetric mode in 30 to 60 seconds since trigger distribution through GCN network, with no optical emission detections. 9 of 250 Fermi GBM triggers have been also followed up in wide-field mode in 20 to 90 seconds from the trigger. All other events were either below the horizon or occurred in bad weather conditions.

\subsection{Simultaneous observations of Fermi GRB 151107B}

The localization of Fermi GBM trigger GRB 151107B (Stanbro \& Meegan 2015; Karpov et al. 2015) has been observed before, during and just after the trigger time, covering nearly all its error box (see Figure 3) simultaneously since $T-329.3 \mathrm{~s}$ till $T+25.7$ (including brightest part of first gamma-ray peak) with temporal resolution of $0.1 \mathrm{~s}$ in white light. Dedicated real-time transient detection pipeline did not detect any events 


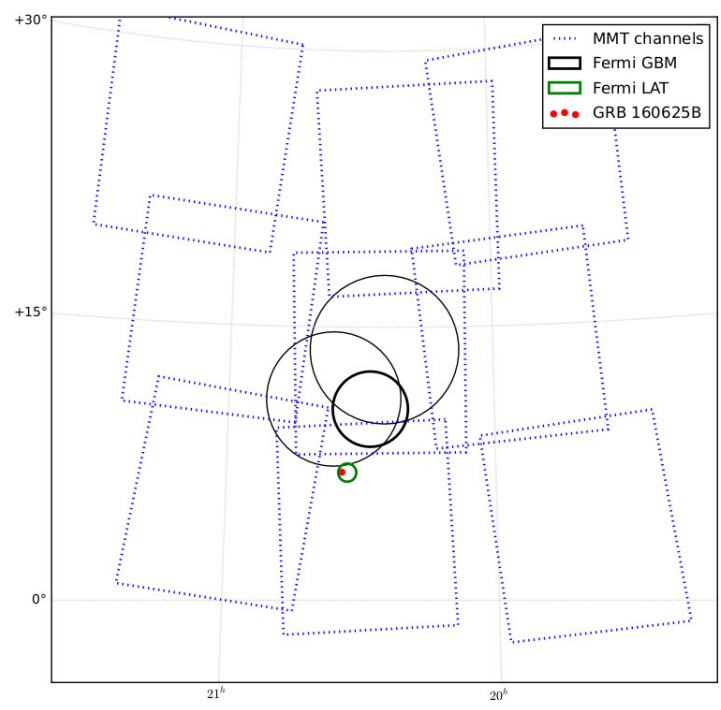

Fig. 4. The same as Figure 3, but for GRB 160625B, with smallest circle representing final LAT error box, and the dot - the localization of optical transient, well outside original 1-sigma circle used for the MiniMegaTORTORA follow-up (9 channels in wide-field regime).

longer than $0.3 \mathrm{~s}$ and brighter than approximately $V=10.5$ mag. Inspection of co-added images with $10 \mathrm{~s}$ effective exposure has not revealed any variable source down to $V=12.0 \mathrm{mag}$ during that interval.

After receiving GCN trigger the system initiated a wide-field follow-up and since $\mathrm{T}+62.7 \mathrm{~s}$ (during the continuing gamma-ray activity) till $T+666.7 \mathrm{~s}$ acquired 20x9 deep images with $30 \mathrm{~s}$ exposures in a 30x30 degree field of view covering the whole final 1sigma localization box. Analysis of the acquired data has not revealed any variable object down to roughly $V=13.5 \mathrm{mag}$ over the time interval (Karpov et al. 2015).

\section{DETECTION OF THE OPTICAL COUNTERPART OF FERMI GRB 160625B}

One more Fermi event, GRB 160625B, has been followed up in the widefield regime, with bright optical flash of GRB 160625B clearly detected during the gamma activity (Karpov et al. 2016, 2017b).

The on-sky position of the Fermi gamma-ray burst GRB 160625B has been observed before, during and just after the LAT trigger time $(T=2016-06$ 25 22:43:24). Mini-MegaTORTORA reacted to the Fermi GBM trigger no. 488587220 generated as a result of the detection of the precursor and started observing its error box 52 seconds after it and 136 seconds before LAT trigger. Due to large size of GBM error box, the observations have been performed in

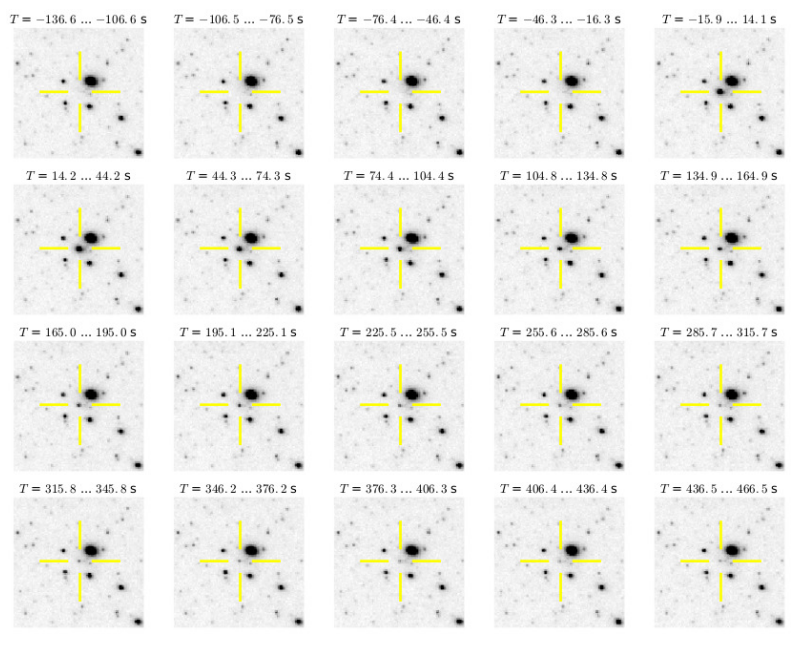

Fig. 5. The final localization region of GRB 160625B as seen by Mini-MegaTORTORA (20 unfiltered images with 30-s exposures). The peak brightness of transient object is $\mathrm{V}=8.8 \mathrm{mag}$.

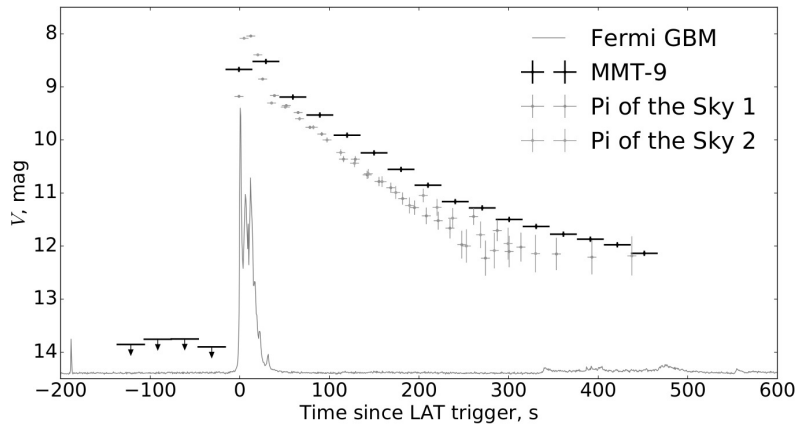

Fig. 6. The light curve of optical transient that accompanied the second gamma activity episode of GRB 160625B, as seen by Mini-MegaTORTORA. Also, the data acquired by Pi of the Sky cameras (Batsch et al. 2016) are shown.

"widefield+deep" regime, with channels simultaneously covering $30 \times 30$ deg field of view (see Figure 4) with $30 \mathrm{~s}$ exposures in white light to achieve deepest detection limit. The system acquired 20 frames in such regime, covering time interval from $T-136$ to $T+466 \mathrm{~s}$, and detected a bright optical transient with a magnitude of about $V=8.8 \mathrm{mag}$ on a frame coincident with LAT trigger time $(T-15.9-$ $T+14.1 \mathrm{~s}$ ) at the coordinates consistent with the afterglow (Troja et al. 2016). On the consecutive frames, the transient brightened for about $0.1 \mathrm{mag}$, and then faded following nearly smooth power-law decay with slope of about -1.6 , down to $V=12.2$ at last acquired frame. The images acquired prior to LAT trigger do not display any object at that posi- 

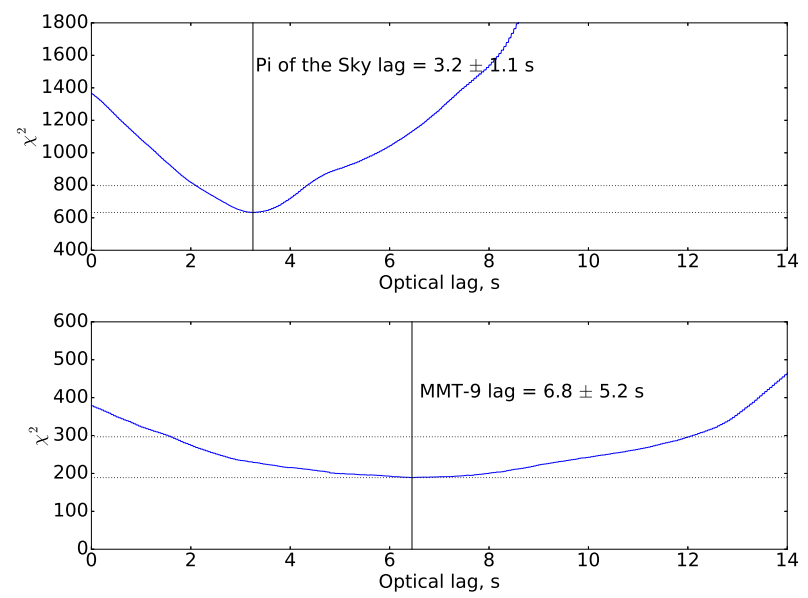

Fig. 7. The determination of a lag between optical and gamma ray light curves of GRB 160625B, using optical measurements from Mini-MegaTORTORA and $\mathrm{Pi}$ of the Sky.

tion down to about $V=13.5 \mathrm{mag}$. This sequence of frames ( $20^{\prime}$ subimages centered on the transient) is shown in Figure 5.

The system also responded to the second GBM trigger no. 488587880 with a somewhat different coordinates. This response resulted in the acquisition of 20 more frames covering burst position in the time interval from $T+1691$ to $T+2264$ s. In this sample no transient objects brighter than $V=13.5 \mathrm{mag}$ were detected.

The optical light curve shown in Figure 6 displays an initial peak with duration similar to the one of the gamma-ray peak and seemingly corresponding to the prompt phase of emission, gradually transforming into the afterglow about $50 \mathrm{~s}$ after the onset of the gamma-ray event. Such a behavior - the absence of the intensity dip between the prompt optical emission accompanying the gamma-ray burst and the afterglow - is typical for several most powerful bursts including GRB 080319B (Naked-Eye Burst) (Beskin et al. 2015). This is not the only similarity between these two events. Indeed, in both cases the intensity of the optical emission accompanying the gamma-ray burst exceeds the extrapolation of the gamma-ray spectrum to the optical range, which indicates different generation mechanisms of these two emission components. Moreover, gamma-ray peaks precede the corresponding optical flashes in time. Indeed, a comparison of the light curves of GRB 160625B in different spectral intervals (see Figure 7 where we used both the Mini-MegaTORTORA data and the results obtained by $\mathrm{Pi}$ of the Sky wide-field monitoring system (Batsch et al. 2016)) shows that optical and gamma-ray emission in the second activity episode are correlated, and the latter precedes the optical flash by 2-4 s. Given the measured redshift of the object, which was found to be close to $1.4(\mathrm{Xu}$ et al. 2016), we find that in the comoving frame optical emission lags behind gamma-ray emission for 1-2 s, like in the Naked-Eye Burst where the same lag was found. We may conclude that in both cases optical photons are born 10-100 times farther away from the "central engine" than high-energy photons, i.e., in jet regions that are spaced apart (Beskin et al. 2010b), and that in the GRB 160625B electrons are heated by internal shocks originating from the residual collisions of filaments ejected in the jet, and the observed emission is generated by their synchrotron energy release (Li \& Waxman 2008).

\section{PHOTOMETRIC ANALYSIS OF MINI-MEGATORTORA DATA}

Most of the Mini-MegaTORTORA observational time since its commissioning in mid-2014 is dedicated to the high temporal resolution (with exposure of $0.1 \mathrm{~s}$ and limit of about $\mathrm{V}=11 \mathrm{mag}$ ) wide-field monitoring of the sky in order to detect and classify rapid optical transients in real time, and to perform their follow-up. In addition to this "monitoring" operation, the system performs deeper "survey" imaging of the sky (one to few frames per night per field with 20 to $60 \mathrm{~s}$ exposure and limit down to $\mathrm{V}=14.5$ mag; more than 300000 frames to date). Moreover, during the monitoring, intermediate depth "running sums" images with $10 \mathrm{~s}$ effective exposures (limit of about $\mathrm{V}=12.5 \mathrm{mag}$ ), amounting typically for 100 consecutive images, spanning 1000 seconds in total, per field, are generated and stored.

\subsection{Detector linearity}

Mini-MegaTORTORA has not been originally designed to be a precise photometric instrument, as it uses fast but less reliable Andor Neo sCMOS detectors in place of more typical and accurate CCDs. Due to it, precise calibration of imaging data from Mini-MegaTORTORA requires thorough study of peculiarities, non-linearity and stability of its detectors.

We performed the linearity analysis of Andor Neo sCMOS cameras installed into MiniMegaTORTORA channels at an interval of bad weather conditions when the observations have been stopped. The channels have been thoroughly isolated from in-dome stray light, and the in-channel flat field LED light sources have been turned on. The intensity of light for every channel has been manually adjusted to be about 300 ADU per pixel per second, averaged over the whole image. This way, by 
varying the exposure time from 0.03 to 60 seconds, we may sample nearly the whole dynamic range of the detectors. To improve the signal to noise ratio, for every exposure we acquired and averaged a series of frames (600 frames for $T_{\text {exp }}<0.2 \mathrm{~s}, 300$ frames for $T_{\text {exp }}<0.5 \mathrm{~s}, 250$ for $T_{\exp }<1.0 \mathrm{~s}, 100$ for $T_{\text {exp }}<2.0 \mathrm{~s}, 40$ for $T_{\text {exp }}<3 \mathrm{~s}, 30$ for $T_{\text {exp }}<5 \mathrm{~s}, 20$ for $T_{\text {exp }}<10 \mathrm{~s}$, and 10 for longer exposures).

To avoid introducing artificial trends related to instabilities of LED light sources power sources, we decided not to vary the exposure times smoothly, but to randomize it using log-uniform distribution. Moreover, after every sequence of frames with this randomized exposures we acquired a set of images with fixed exposure time (1 second). This way, we were able to accurately track the smooth changes in light source intensity and correct for it.

The values $I$ for every pixel have been then fitted by a linear function $I=a \cdot T_{\text {exp }}+b$, where $b$ represents bias value, which is pixel-dependent due to CMOS pixel architecture, and $a$ - both per-pixel gain and sensitivity, and the spatially-varying light intensity. The deviations from this simple linear regression represents the non-linearity of the detector. Example of such non-linearity is shown in upper panel of Figure 8. One may easily identify at least four distinct regions there, most striking being the jump-like feature at around $1400 \mathrm{ADU}$ in global shutter and 1700 ADU in rolling shutter with amplitude up to several percents, caused by the transition from the high gain to low gain amplifiers in dual-amplifier sCMOS design (Andor Technology Ltd. 2012). The properties of these regions vary from pixel to pixel. To calibrate it, we fitted these dependencies with a piecewise fourth order polynomial with connection point varying as an additional parameter. After applying such correction, we are able to linearize the detector to a level of about $1 \%$ over the whole dynamic range nearly up to saturation limit (see Figure 8).

\subsection{Photometric pipeline}

In general, the archive of Mini-MegaTORTORA images represents a time-domain picture of the sky on time scales ranging from tens of seconds to years, with hundreds to tens of thousands points for every object, and in principle allows to extract and characterize the majority of variable objects down to $\mathrm{V}=14.5 \mathrm{mag}$.

The data in the archive are first corrected for the non-linearity using optimal parameters measured for every pixel of every detector in a way desctibed in Section 4.1. Then the usual bias and dark subtraction is performed, followed by a flat-fielding us-
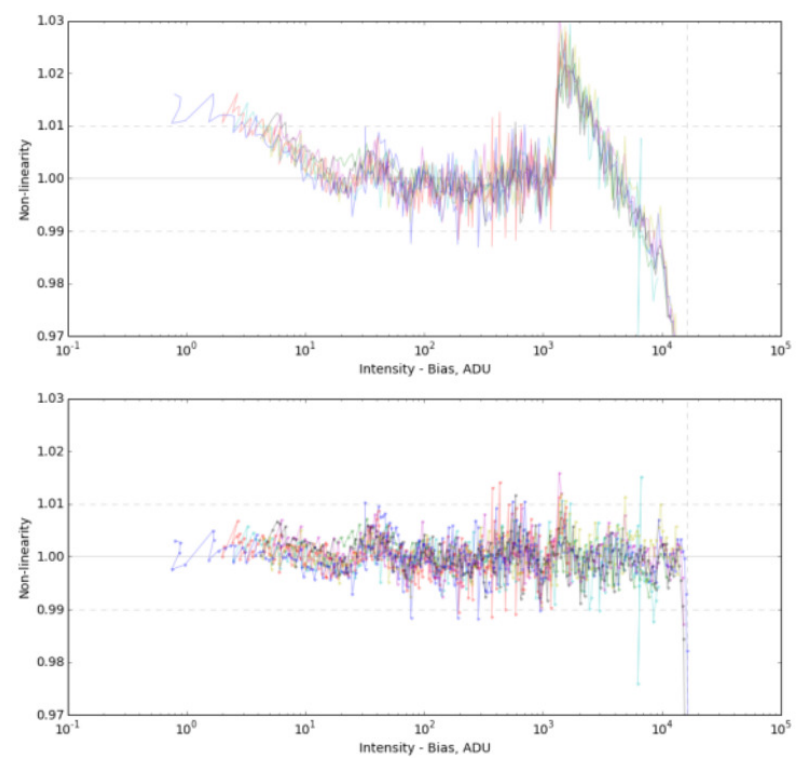

Fig. 8. Top - non-linearity of Andor Neo sCMOS detector in global shutter mode as a function of intensity due to its dual-amplifier design, shown for a random sample of pixels. Bottom - the same after applying linearization correction defined as a piecewise fourth-order polynomial with parameters calibrated on a per-pixel basis.

ing the master flat frames computed by either acquiring evening sky images or median averaging of a frames of the stellar fields over large time intervals and after masking of stars and artefacts in the frames. Both methods of deriving master flats are acceptable as they allow to compensate for a smallscale non-uniformities of the CMOS structure, as well as dust on the detectors, lenses and coelostat mirrors. Larger scale non-uniformities and gradients are accounted later, during the photometric calibration stage.

The detection and photometry of the stars on every frame is done using dedicated pipeline built around SEP library (Barbary 2016), which is a reusable Python interface to some of SExtractor (Bertin \& Arnouts 1996) low-level algorithms. We use it for both detection, which is performed after applying some masking heuristics which exclude parts of image covered by clouds, bright artificial satellite tracks and other image artifacts, as well as badly behaving CMOS pixels, and for aperture photometry using simple circular apertures with optimal sizes based on mean FWHM over the image.

In quite large fraction of survey images, especially acquired at low Galactic latitudes, the stellar fields are significantly crowded due to large pixel size of the detector we use, as well as imperfect image quality of Canon EF85/1.2 objective, which is producing 


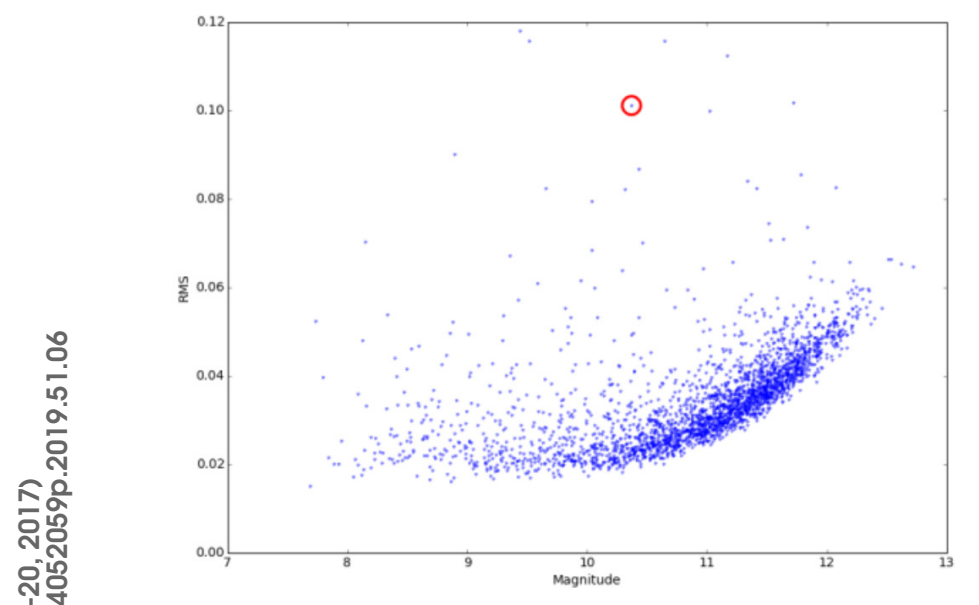

Fig. 9. Example of a scatter versus mean magnitude plot for a sky region. Red cicrle marks the variable star detected by our pipeline, which is absent from AAVSO VSX database (see Figure 10).

significant and widely spread PSF wings. Therefore we also implemented an in-house PSF photometry code which handles both matched filter detection, extraction, profile-fitting photometry and iterative deblending of the point-like sources. However, due to some shortcomings in the PSFEx (Bertin 2011) code we use for actual PSF consruction, we are not using this code regularly yet.

Next, the measurements from every frame are calibrated using Tycho-2 catalogue (Høg et al. 2000) which is still the only exoatmospheric survey covering the whole sky in our range of magnitudes. As all Mini-MegaTORTORA survey frames are acquired in white light, the instrumental magnitudes $m_{\text {instr }}$ are fitted against linear combination of $B_{T}$ and $V_{T}$ magnitudes with coefficients fixed to the ones optimized over the whole survey:

$$
m_{\text {instr }}=m_{0}(x, y)+0.37 B_{T}-0.45 V_{T}
$$

where magnitude zero point $m_{0}(x, y)$ is a fourth order spatially varying polynomial accounting both for atmospheric extinction and uncorrected gradients left after flatfield correction. The calibrated magnitudes are stored to the PostgreSQL database and are indexed using Q3C spherical indexing scheme Koposov \& Bartunov (2006) for performing fast positional queries.

Finally, in order to search for variability of some sky field, all the measurements from this region are extracted from database and clustered using spherical distances to form measurement groups corresponding to isolated objects. Then for every object the mean magnitude is computed and the spread of measurements around this value is estimated.
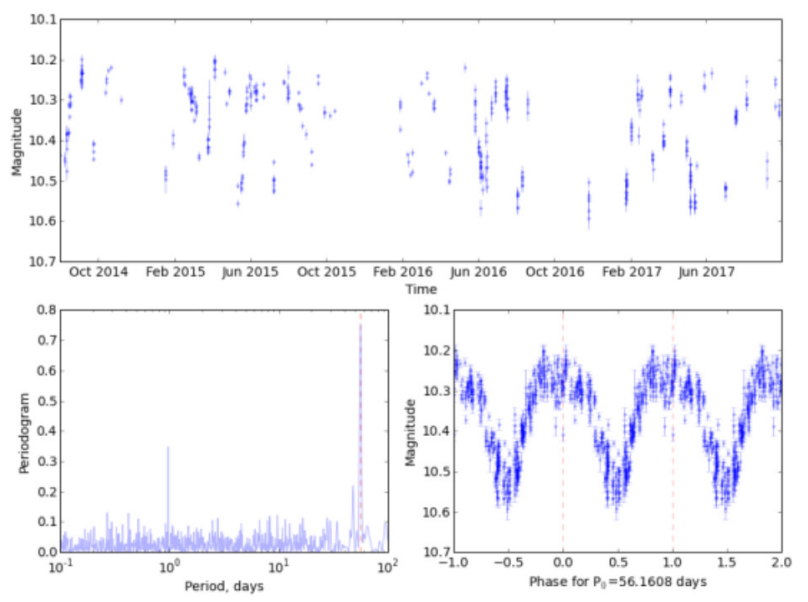

Fig. 10. Example of a new variable star detected in the Mini-MegaTORTORA data. Top panel - light curve, bottom - periodogram and a folded light curve corresponding to the best period detected in periodigram.

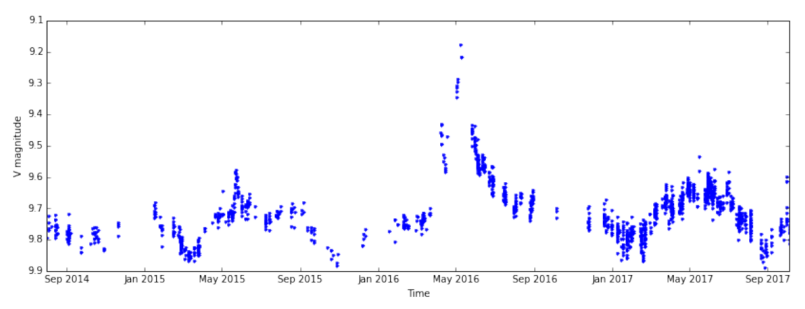

Fig. 11. Light curve of AG Dra symbiotic binary star extracted from "survey" images stored in MiniMegaTORTORA data archive. The large outburst around May 2016 is clearly visible, along with some smaller ones before and after.

Then, after excluding stars with large scatter, we re-calibrate all the frames covering the region using mean magnitudes for stars instead of Tycho-2 ones, and repeat the process until the values are converged and overall scatter of magnitude values are minimized for all objects. Then we build scatter versus magnitude plots (see Figure 9), select objects with large scatter, and perform periodicity search for them. Examples of variable stars from MiniMegaTORTORA archive are shown in Figures 10 and 11.

The analysis of "running sum" images is performed in a similar way except for the measurements are not stored to the database due to huge amount of data available. Instead, every sequence (which is typically 100 frames long) is processed independently in the same two-stage manner, first pass to match brighter stars against Tycho-2 catalogue (Høg et al. 2000), and the second to derive mean magnitudes and re-fit all the frames using them as an input catalogue. Unfortunately, all the "running sum" data 

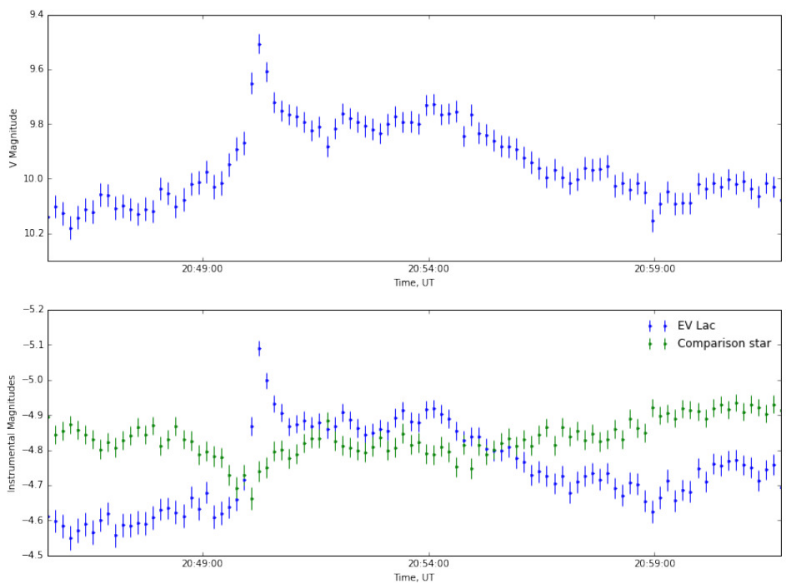

Fig. 12. Light curve of EV Lac flaring star extracted from "running sum" frames (10 s effective exposure, derived by averaging 100 consecutive frames acquired in monitoring) in Mini-MegaTORTORA archive. The unfiltered instrumental magnitudes (lower panel) are calibrated to $\mathrm{V}$ band (upper panel) by using the comparison star of similar color to the object. The noise in the frames is estimated as a spatial variance and is mostly due to $\mathrm{sC}$ MOS non-uniform bias map. Instrumental magnitudes demonstrate some trends due to drifting of pixel bias values and slow motion of the stars over different pixels due to imperfect tracking.

have very low signal level, and the effects of detector non-linearity and badly behaving CMOS pixels are the strongest for them. Due to it, the resulting photometric data are contaminated by artificial trends caused by star motion over the detector due to imperfect tracking. Such trends can't be easily filtered out by ensemble photometry as they are mostly pixel-based. Therefore, we are not currently employing these data to look for slowly-variable objects. However, we are performing the flare search in it in order to detect and characterize flaring stars, which may then be observed on larger telescopes (Beskin et al. 2017a,b). Figure 12 shows the example of a strong flare detected on EV Lac red dwarf star.

\section{CONCLUSIONS}

Over three years of its operation, the MiniMegaTORTORA instrument has demonstrated its potential for studying various phenomena on the sky, both astrophysical and artificial in origin. Its on-line databases and survey data may be used for studying faint meteoric streams crossing Earth orbit, for detecting new comets and asteroids, for finding flashes of flaring stars and novae, studying variable stars of various classes, detecting transits of exoplanets, searching for bright supernovae and optical counterparts of gamma-ray bursts.
Acknowledgements: Mini-MegaTORTORA belongs to Kazan Federal University and the work is performed according to the Russian Government Program of Competitive Growth of Kazan Federal University. Observations on Mini-MegaTORTORA are supported by the Russian Science Foundation grant No. 14-50-00043. The reported study was funded by RFBR according to the research project No. 17-52-45048. This work was supported by European Structural and Investment Fund and the Czech Ministry of Education, Youth and Sports (Project CoGraDS - CZ.02.1.01/0.0/0.0/15_003/0000437).

\section{REFERENCES}

Andor Technology Ltd. 2012, Dual Amplifier Dynamic Range, http://www.andor.com/learning-academy/ dual-amplifier-dynamic-range-scmos-dynamic7 -range

Barbary, K. 2016, JOSS, 1, 58

Batsch, T., Castro-Tirado, A. J., Czyrkowski, H., Cwiek, A., Cwiok, M., et al. 2016, GCN, 19615

Bertin, E. 2011, ASPC 442, Astronomical Data Analysis Software and Systems XX, ed. I. N. Evans, A. Accomazzi, D. J. Mink, \& A. H. Rots, 435

Bertin, E. \& Arnouts, S. 1996, A\&A, 117, 393

Beskin, G., Biryukov, A., Bondar, S., Hurley, K., Ivanov, E., et al. 2004, AN, 325, 676

Beskin, G., Bondar, S., Karpov, S., Plokhotnichenko, V., Guarnieri, A., et al. 2010a, AdAst, 2010, 53

Beskin, G., Karpov, S., Bondar, S., Greco, G., Guarnieri, A., et al. 2010b, ApJ, 719, L10

Beskin, G., Karpov, S., Bondar, S., Perkov, A., Ivanov, E., et al. 2014, RMxAC, 45, 20

Beskin, G., Karpov, S., Plokhotnichenko, V., Stepanov, A., \& Tsap, Y. 2017a, PASA, 34, 10

Beskin, G., Karpov, S., Plokhotnichenko, V., Stepanov, A., \& Tsap, Y. 2017b, ASPC 510, 303

Beskin, G. M., Karpov, S. V., Biryukov, A. V., Bondar, S. F., Ivanov, E. A., et al. 2017c, AstBu, 72, 81

Beskin, G. M., Karpov, S. V., Bondar, S. F., Plokhotnichenko, V. L., Guarnieri, A., et al. 2010c, PhyU, 53, 406

Beskin, G. M., Karpov, S. V., Plokhotnichenko, V. L., Bondar, S. F., Perkov, A. V., et al. 2013, PhyU, 56, 836

Beskin, G. M., Oganesyan, G., Greco, G., \& Karpov, S. 2015, AstBu, 70, 400

Høg, E., Fabricius, C., Makarov, V. V., Urban, S., Corbin, T., et al. 2000, A\&A, 355, L27

Karpov, S., Beskin, G., Biryukov, A., Bondar, S., Hurley, K., et al. 2005, NCimC, 28, 747

Karpov, S., Beskin, G., Biryukov, A., Bondar, S., Ivanov, E., et al. 2017a, ASPC 510, 526

Karpov, S., Beskin, G., Biryukov, A., Bondar, S., Ivanov, E., et al. 2017b, ASPC 510, 309 
Karpov, S., Beskin, G., Bondar, S., Guarnieri, A., Bartolini, C., et al. 2010, AdAst, 2010, 40

Karpov, S., Beskin, G., Bondar, S., Ivanov, E., Katkova, E., et al. 2016, GCN, 19603

Karpov, S., Beskin, G., Bondar, S., Ivanov, E., Katkova, E., et al. 2015, GCN, 18574

Koposov, S. \& Bartunov, O. 2006, ASPC 351, 735

Lang, D., Hogg, D. W., Mierle, K., Blanton, M., \& Roweis, S. 2010, AJ, 139, 1782

Li, Z. \& Waxman, E. 2008, ApJ, 674, 65
Molinari, E., Bondar, S., Karpov, S., Beskin, G., Biryukov, A., et al. 2006, NCimB, 121, 1525

Stanbro, M. \& Meegan, C. 2015, GCN, 18570

Troja, E., Butler, N., Watson, A. M., Kutyrev, A., Lee, W. H., et al. 2016, GCN, 19588

Xu, D., Malesani, D., Fynbo, J. P. U., Tanvir, N. R., Levan, A. J., et al. 2016, GCN, 19600

Zolotukhin, I., Beskin, G., Biryukov, A., Bondar, S., Hurley, K., et al. 2004, AN, 325, 675 